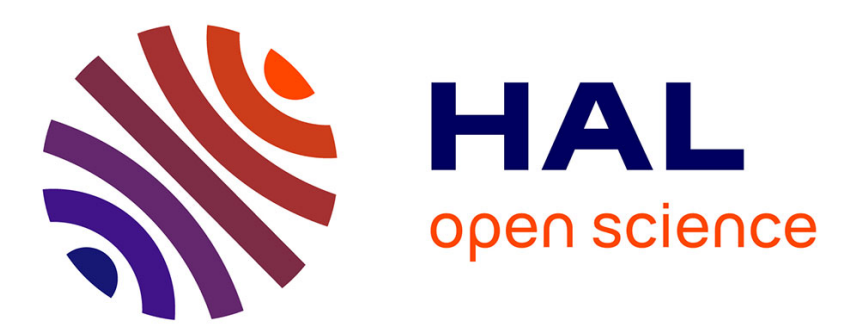

\title{
Effect of disorder on two-dimensional wetting
}

Bernard Derrida, V. Hakim, J. Vannimenus

\section{To cite this version:}

Bernard Derrida, V. Hakim, J. Vannimenus. Effect of disorder on two-dimensional wetting. Journal of Statistical Physics, 1992, 66 (5-6), pp.1189-1213. 10.1007/BF01054419 . hal-03282960

\section{HAL Id: hal-03282960 https://hal.science/hal-03282960}

Submitted on 19 Jul 2021

HAL is a multi-disciplinary open access archive for the deposit and dissemination of scientific research documents, whether they are published or not. The documents may come from teaching and research institutions in France or abroad, or from public or private research centers.
L'archive ouverte pluridisciplinaire HAL, est destinée au dépôt et à la diffusion de documents scientifiques de niveau recherche, publiés ou non, émanant des établissements d'enseignement et de recherche français ou étrangers, des laboratoires publics ou privés. 


\title{
Effect of Disorder on Two-Dimensional Wetting
}

\author{
B. Derrida, ${ }^{1}$ V. Hakim, ${ }^{2}$ and J. Vannimenus ${ }^{2}$
}

Received September 11, 1991

\begin{abstract}
For the problem of the $2 \mathrm{D}$ wetting transition near a $1 \mathrm{D}$ random wall, we show by a renormalization calculation that the effect of disorder is marginally relevant. It is therefore expected that the nature of the wetting transition and the location of the critical point are modified by any amount of disorder. This is supported by numerical simulations based on transfer matrix calculations. We investigate also the problem of wetting near a random wall on hierarchical lattices and find similar results.
\end{abstract}

KEY WORDS: Wetting transition; disorder; transfer matrix.

\section{INTRODUCTION}

An important problem in the theory of disordered systems is to understand the effect of a small concentration of impurities on the nature of a phase transition. ${ }^{(1,2)}$ For magnetic systems, when the effect of the impurities can be represented by random exchange interactions, one knows, from the Harris criterion, ${ }^{(1)}$ that a weak disorder changes the nature of a secondorder phase transition only if the specific heat exponent $\alpha$ of the pure system is positive. For negative $\alpha$, disorder is irrelevant, meaning that a weak enough disorder leaves the critical exponents characteristic of the phase transition unchanged. The case $\alpha=0$ is, as usual in the renormalization group approach, marginal and one needs to know the sign of the nonlinear term in the renormalization flow to decide whether disorder is marginally relevant or irrelevant.

\footnotetext{
${ }^{1}$ Service de Physique Théorique de Saclay (Laboratoire de la Direction des Sciences de la Matière du Commissariat à l'Energie Atomique), F-91191 Gif-sur-Yvette Cedex, France.

${ }^{2}$ Laboratoire de Physique Statistique (Laboratoire associé au CNRS et aux Universités Paris 6 et Paris 7), ENS, 24 rue Lhomond, 75231 Paris Cedex 05, France.
} 
The purpose of the present paper is to discuss this question in one of the simplest examples of a phase transition, the problem of wetting ${ }^{(3-5)}$ near a random one-dimensional wall.

In its simplest version, the problem of wetting is that of an interface attracted by a wall. At low enough temperature, the interface is localized near the wall and it becomes free at the wetting transition.

Here we shall consider the following SOS version of the wetting problem near a random surface. ${ }^{(6,7)} \mathrm{A}$ configuration of the interface is represented by a sequence of integer heights $h_{i} \geqslant 0$ which measure the distance between the interface and the wall at abscissa $i$. By definition of the model, the heights $h_{i}$ at neighboring sites can differ at most by 1 ,

$$
h_{i+1}-h_{i}=-1,0, \text { or }+1
$$

At each site $i$, there is a random energy $-u_{i}$ if the configuration touches the wall, i.e., if $h_{i}=0$. There is an additional energy $J$ associated to each step between consecutive heights. Therefore, the energy $E\left(\left\{h_{i}\right\}\right)$ of a configuration is

$$
E\left(\left\{h_{i}\right\}\right)=J \sum_{i}\left|h_{i}-h_{i+1}\right|-\sum_{i} u_{i} \delta_{h_{i}, 0}
$$

If one defines $Z_{L}(x, y)$ as the partition function of an interface pinned at height $h_{0}=x$ and $h_{L}=y$, one can write the following recursion:

$$
Z_{L+1}(x, y)=Z_{L}(x, y)+t\left[Z_{L}(x, y+1)+Z_{L}(x, y-1)\right] \quad \text { for } y \geqslant 1
$$

and

$$
Z_{L+1}(x, 0)=a_{L+1}\left[Z_{L}(x, 0)+t Z_{L}(x, 1)\right]
$$

with

$$
Z_{0}(x, y)=\delta_{x, y}
$$

where the parameters $a_{i}$ and $t$ are defined by

$$
a_{i}=e^{u_{i} / T} \quad \text { and } \quad t=e^{-J / T}
$$

( $T$ is the temperature).

In what follows, we shall consider $t$ as a fixed parameter (we will forget its temperature dependence) and we shall write

$$
a_{i}=\langle a\rangle\left(1+b_{i}\right)
$$


where the $b_{i}$ are random numbers equally distributed with a zero average

$$
\left\langle b_{i}\right\rangle=0
$$

Forgacs et al ${ }^{(6,7)}$ considered this problem in the weak-disorder limit $\left\langle b^{2}\right\rangle \ll 1$. They found that disorder is marginal at the wetting transition of the pure system given by $\langle a\rangle=(1+2 t) /(1+t)$, because the specific heat exponent $\alpha=0$. Moreover, they suggested that disorder is marginally irrelevant. $^{(6,7)}$ By resumming the leading diagrams of a weak-disorder expansion, Forgacs et al. ${ }^{(6,7)}$ concluded that for a weak enough disorder, the transition temperatures of the quenched and annealed models are equal [they occur when $\left.\langle a\rangle=\langle a\rangle_{c}^{\text {pure }}=(1+2 t) /(1+t)\right]$. They also concluded that the critical behavior at the transition is not changed by disorder except for a subdominant $1 / \log$ term in the specific heat.

In the present paper, we show by a renormalization group calculation that disorder is marginally relevant. This is in contrast with another wellstudied marginal case, ${ }^{(8,9)}$ the $2 \mathrm{D}$ Ising model for which disorder is marginally irrelevant, implying that the critical behavior of the pure and random cases are identical except for logarithmic corrections. Because disorder is relevant, we expect the critical behavior at the transition to be modified by any amount of disorder. But because under renormalization the system flows toward strong-disorder regions, where perturbative approaches are useless, we cannot predict the nature of the phase transition. Also, the critical value $\langle a\rangle_{c}^{\text {random }}$ has no reason to remain unchanged by disorder and we expect

$$
\log \left(\langle a\rangle_{c}^{\text {random }}-\langle a\rangle_{c}^{\text {pure }}\right) \simeq-\frac{\pi t(1+2 t)}{2(1+t)^{2}\left\langle b^{2}\right\rangle}
$$

when $\left\langle b^{2}\right\rangle \rightarrow 0$.

This is indeed the general shape of an arbitrary manifold near the pure fixed point and there is no reason for the critical manifold to be exactly $\langle a\rangle_{c}^{\text {random }}=\langle a\rangle_{c}^{\text {pure }}$. This prediction (1.7) does not contradict the result ${ }^{(6,7)}$ that the transition temperatures of the annealed and the quenched models coincide to all orders in the weak-disorder expansion.

The paper is organized as follows: we first show (Section 2) by a renormalization group calculation that disorder is marginally relevant at the wetting transition in dimension two.

In Section 3 we present the results of some numerical studies where one can see the shift in the transition temperature and that the specific heat vanishes continuously at the transition in the presence of disorder (instead of a jump in the pure case).

In Section 4, we formulate the problem of wetting in the presence of 
disorder on a hierarchical lattice. On such lattices, the approach is simpler and numerical studies can be done with better accuracy. In that case, too, we find that disorder is marginally relevant and numerical simulations indicate a shift in the transition temperature and a specific heat which vanishes at the transition.

\section{THE WETTING PROBLEM NEAR A 1D RANDOM SUBSTRATE}

To discuss the effect of disorder, we follow here an approach which is, in spirit, an exact real space renormalization.

\subsection{Recursion Relations}

Our starting point is a recursion relation for the partition function $Z_{L}(x, y)$ of an interface of length $L$, the end points of which are pinned at heights $x$ at abscissa 0 and $y$ at abscissa $L$ (i.e., $h_{0}=x$ and $h_{L}=y$ ). This partition function $Z_{L}$ satisfies the following recursion relation (Fig. 1):

$$
Z_{2 L}(x, y)=\sum_{z \geqslant 0} Z_{L}^{(1)}(x, z) Z_{L}^{(2)}(z, y)
$$

where $Z_{L}^{(1)}(x, z)$ represents the sum over all interfaces of length $L$ starting at height $x$ at abscissa 0 and ending at height $z$ at abscissa $L$, and $Z_{L}^{(2)}(z, y)$ represents all the interfaces starting at height $z$ at abscissa $L$ and ending at height $y$ at abscissa $2 L$. The origin of the recursion (2.1) is just the fact that each interface of length $2 L$ can be decomposed into two pieces of length $L$. Because the wall is inhomogeneous, $Z_{L}^{(1)}$ and $Z_{L}^{(2)}$ are not identical. Each of them is random since it depends on the random wall potential, but they are independent because $Z_{L}^{(1)}$ depends only on the potential between 1 and $L$, and $Z_{L}^{(2)}$ on the potential between $L+1$ and $2 L$ [here disorder on the substrate is chosen to be uncorrelated and by convention in $Z_{L}(x, y)$ we count all the weights due to the contacts from abscissa 1 to $L]$. .

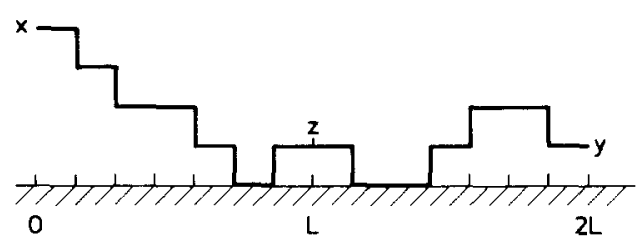

Fig. 1. A configuration of the interface in the SOS model. The hatched surface represents the attractive wall. 
To study the system near the wetting transition, it is convenient to make the following change of variables:

$$
Y_{L}(u, v)=(2+2 t)^{-L} \sqrt{L_{0}} Z_{L}(x, y)
$$

where

$$
u=x / \sqrt{L_{0}} ; \quad v=y / \sqrt{L_{0}} ; \quad L_{0}=\frac{4 t}{1+2 t} L
$$

For large $L, u$ and $v$ become continuous variables and the recursion (2.1) becomes

$$
Y_{2 L}\left(\frac{u}{\sqrt{2}}, \frac{v}{\sqrt{2}}\right)=\sqrt{2} \int_{0}^{\infty} d w Y_{L}^{(1)}(u, w) Y_{L}^{(2)}(w, v)
$$

The partition function $Z_{L}(x, y)$ of the random system is given to first order in disorder by

$$
Z_{L}(x, y)=Z_{L}^{\text {pure }}(x, y)+\sum_{k=1}^{L} b_{k} Z_{k}^{\text {pure }}(x, 0) Z_{L-k}^{\text {pure }}(0, y)
$$

where $Z_{L}^{\text {pure }}$ denotes the partition function in the pure case, i.e., when all the $b_{i}=0$.

At the wetting transition of the pure system $[\langle a\rangle=(1+2 t) /(1+t)]$, one can show that (Appendix A) for $x \geqslant 1$

$$
\begin{aligned}
Z_{L}^{\text {pure }}(x, y) \simeq & (1+2 t)^{L}\left(\frac{1+2 t}{4 t \pi L}\right)^{1 / 2}\left[\exp \left(-\frac{(x-y)^{2}(1+2 t)}{4 t L}\right)\right. \\
& \left.+\exp \left(-\frac{(x+y)^{2}(1+2 t)}{4 t L}\right)\right]
\end{aligned}
$$

whereas for $x=0$

$$
Z_{L}^{\text {pure }}(0, y) \simeq(1+2 t)^{L}\left(\frac{1+2 t}{4 t \pi L}\right)^{1 / 2} \frac{1+t}{1+2 t} 2 \exp \left(-\frac{y^{2}(1+2 t)}{4 t L}\right)
$$

The expression for $x=0$ is slightly different because in $Z_{L}(x, y)$ we count all the weights due to the contacts with the wall from abscissa 1 to abscissa $L$. [Notice also that due to this convention, $Z_{L}(x, 0)=$ $Z_{L}(0, x)(1+2 t) /(1+t)$ for $x \geqslant 1$, whereas $Z_{L}(x, y)=Z_{L}(y, x)$ otherwise. $]$

Equations (2.6) imply through (2.2) and (2.3) that

$$
Y_{L}(u, v)=Y_{L}^{\text {pure }}(u, v)+g_{L}(u, v)
$$


where

$$
Y_{L}^{\text {pure }}(u, v)=\frac{1}{\sqrt{\pi}}\left[e^{-(u-v)^{2}}+e^{-(u+v)^{2}}\right]
$$

and

$$
\begin{aligned}
g_{L}(u, v)= & \sum_{k=1}^{L}\left(\frac{1}{[(k / L)(1-k / L)]^{1 / 2}}\right) \frac{4}{\sqrt{L_{0}}} b_{k} \frac{1+t}{1+2 t} \frac{1}{\pi} \\
& \times \exp \left(-\frac{u^{2} L}{k}-\frac{v^{2}}{1-k / L}\right)
\end{aligned}
$$

If one replaces $Y_{L}^{(1)}$ and $Y_{L}^{(2)}$ by their expressions in the recursion (2.4), one finds that $Y_{L}^{\text {pure }}(u, v)$ is a fixed point of this recursion (the critical fixed point) and that the perturbation $g_{L}(u, v)$ satisfies

$$
\begin{aligned}
g_{2 L}\left(\frac{u}{\sqrt{2}}, \frac{v}{\sqrt{2}}\right)= & \frac{\sqrt{2}}{\sqrt{\pi}} \int_{0}^{\infty} d w\left[e^{-(u-w)^{2}}+e^{-(u+w)^{2}}\right] g_{L}^{(2)}(w, v) \\
& +\frac{\sqrt{2}}{\sqrt{\pi}} \int_{0}^{\infty} d w\left[e^{-(w-v)^{2}}+e^{-(w+v)^{2}}\right] g_{L}^{(1)}(u, w) \\
& +\sqrt{2} \int_{0}^{\infty} d w g_{L}^{(1)}(u, w) g_{L}^{(2)}(w, v)
\end{aligned}
$$

This expression is a direct consequence of (2.1) and (2.4) and therefore is exact in the scaling limit $[L$ large, $\langle a\rangle=(1+2 t) /(1+t)]$. The question of whether disorder is relevant or not is simply to know if $g_{L}$ grows under the renormalization (2.10). It is easier to investigate this question by using the Fourier transform of $g_{L}(u, v)$,

$$
G_{L}\left(K_{1}, K_{2}\right)=\frac{1}{(2 \pi)^{2}} \int_{-\infty}^{+\infty} \int_{-\infty}^{+\infty} d u d v e^{-i K_{1} u-i K_{2} v} g_{L}(u, v)
$$

[Since $g_{L}(u, v)$ is only defined for positive $u$ and $v$, we can extend the definition to all $u$ and $v$ by choosing $g_{L}(u, v)=g_{L}(|u|,|v|)$. $]$ For the $G_{L}$, the recursion (2.10) becomes

$$
\begin{aligned}
G_{2 L}\left(K_{1}, K_{2}\right)= & \frac{1}{\sqrt{2}}\left[\exp \left(-\frac{K_{1}^{2}}{8}\right) G_{L}^{(2)}\left(\frac{K_{1}}{\sqrt{2}}, \frac{K_{2}}{\sqrt{2}}\right)\right. \\
& \left.+\exp \left(-\frac{K_{2}^{2}}{8}\right) G_{L}^{(1)}\left(\frac{K_{1}}{\sqrt{2}}, \frac{K_{2}}{\sqrt{2}}\right)\right] \\
& +\frac{\pi}{\sqrt{2}} \int d q G_{L}^{(1)}\left(\frac{K_{1}}{\sqrt{2}}, q\right) G_{L}^{(2)}\left(-q, \frac{K_{2}}{\sqrt{2}}\right)
\end{aligned}
$$




\subsection{The Uniform Substrate}

Let us first consider the pure case, i.e., when $G_{L}^{(1)}$ and $G_{L}^{(2)}$ are equal. Then if one linearizes the renormalization transformation (2.12), one gets

$$
G_{2 L}\left(K_{1}, K_{2}\right)=\frac{1}{\sqrt{2}}\left[\exp \left(-\frac{K_{1}^{2}}{8}\right)+\exp \left(-\frac{K_{2}^{2}}{8}\right)\right] G_{L}\left(\frac{K_{1}}{\sqrt{2}}, \frac{K_{2}}{\sqrt{2}}\right)
$$

Since in the pure case, all the $b_{k}$ are equal $\left(b_{k}=b\right)$, one gets from (2.9) and (2.11)

$$
G_{L}\left(K_{1}, K_{2}\right)=\frac{2 b \sqrt{L}}{\pi^{2}} \frac{1+t}{[t(1+2 t)]^{1 / 2}} \frac{\exp \left(-K_{1}^{2} / 4\right)-\exp \left(-K_{2}^{2} / 4\right)}{K_{2}^{2}-K_{1}^{2}}
$$

This turns out to be a solution of the recursion (2.13). We see that the coefficient of $G_{L}\left(K_{1}, K_{2}\right)$ given in (2.14) is a relevant scaling field with dimension $1 / 2$ since as $L$ increases by a factor $2, G_{L}$ increases by a factor $\sqrt{2}$. Thus a system of size $2 L$ at a distance $\Delta T$ from the wetting transition behaves like a system of size $L$ at a distance $\sqrt{2} \Delta T$ from $T_{c}$. One can notice from (2.13) that there are other solutions of (2.13):

$$
G_{L}\left(K_{1}, K_{2}\right)=L^{(1-n) / 2} \frac{\exp \left(-K_{1}^{2} / 4\right)-\exp \left(-K_{2}^{2} / 4\right)}{K_{2}^{2}-K_{1}^{2}} P_{n}\left(K_{1}, K_{2}\right)
$$

where $P_{n}\left(K_{1}, K_{2}\right)$ is an arbitrary homogeneous polynomial of degree $n$. Only $n=0$ corresponds to a relevant scaling field. Since the Fourier transform of a perturbation $g(u, v)$ localized around the origin $u=0, v=0$ can be decomposed on the basis of the functions (2.15), we see that the component on the function (2.14) will be the only one to increase with $L$, meaning that $(2.14)$ is the only relevant scaling field.

\subsection{The Random Substrate}

Let us now consider a perturbation $g_{L}(u, v)$ as given by (2.9) where the $b_{k}$ are random and independent, with $\left\langle b_{k}\right\rangle=0$.

Clearly, one has

$$
\left\langle g_{L}(u, v)\right\rangle=0
$$

and to leading order in the disorder, (2.9) implies

$$
\begin{aligned}
\left\langle g_{L}(u, v) g_{L}\left(u^{\prime}, v^{\prime}\right)\right\rangle= & \frac{\left\langle b^{2}\right\rangle}{\pi^{2}} \frac{(1+t)^{2}}{t(1+2 t)} \\
& \times 4 \int_{0}^{1} d x \frac{\exp \left[-\left(u^{2}+u^{\prime 2}\right) / x-\left(v^{2}+v^{\prime 2}\right) /(1-x)\right]}{x(1-x)}
\end{aligned}
$$


Here again it is more convenient to deal with the Fourier transforms. From (2.17), one gets

$$
\begin{aligned}
& \left\langle G_{L}\left(K_{1}, K_{2}\right) G_{L}\left(K_{3}, K_{4}\right)\right\rangle \\
& \quad=\frac{\left\langle b^{2}\right\rangle}{\pi^{4}} \frac{(1+t)^{2}}{t(1+2 t)} \frac{\exp -\left(K_{1}^{2}+K_{3}^{2}\right) / 4-\exp -\left(K_{2}^{2}+K_{4}^{2}\right) / 4}{K_{2}^{2}+K_{4}-K_{1}^{2}-K_{3}^{2}}
\end{aligned}
$$

Knowing that $\left\langle G_{L}\left(K_{1}, K_{2}\right) G_{L}\left(K_{3}, K_{4}\right)\right\rangle$ is given by (2.18) for a very weak disorder, the question is to know if these correlations will increase when one increases $L$. From the recursion relation (2.12), it follows that

$$
\begin{aligned}
\left\langle G_{2 L}\right. & \left.\left(K_{1}, K_{2}\right) G_{2 L}\left(K_{3}, K_{4}\right)\right\rangle \\
= & \left\langle G_{L}\left(\frac{K_{1}}{\sqrt{2}}, \frac{K_{2}}{\sqrt{2}}\right) G_{L}\left(\frac{K_{3}}{\sqrt{2}}, \frac{K_{4}}{\sqrt{2}}\right)\right\rangle \frac{1}{2}\left(\exp -\frac{K_{1}^{2}+K_{3}^{2}}{8}+\exp -\frac{K_{2}^{2}+K_{4}^{2}}{8}\right) \\
& +\frac{\pi^{2}}{2} \iint d q_{1} d q_{2}\left\langle G_{L}\left(\frac{K_{1}}{\sqrt{2}}, q_{1}\right) G_{L}\left(\frac{K_{3}}{\sqrt{2}}, q_{2}\right)\right\rangle \\
& \times\left\langle G_{L}\left(-q_{1}, \frac{K_{2}}{\sqrt{2}}\right) G_{L}\left(-q_{2}, \frac{K_{4}}{\sqrt{2}}\right)\right\rangle
\end{aligned}
$$

As in the pure case, one can look first at the linear part of (2.19) and one finds solutions of the form

$$
\begin{aligned}
& \left\langle G_{L}\left(K_{1}, K_{2}\right) G_{L}\left(K_{3}, K_{4}\right)\right\rangle \\
& \quad=L^{-n / 2} \frac{\exp -\left(K_{1}^{2}+K_{3}^{2}\right) / 4-\exp -\left(K_{2}^{2}+K_{4}^{2}\right) / 4}{K_{2}^{2}+K_{4}^{2}-K_{1}^{2}-K_{3}^{2}} P_{n}\left(K_{1}, K_{2}, K_{3}, K_{4}\right)
\end{aligned}
$$

where $P_{n}\left(K_{1}, K_{2}, K_{3}, K_{4}\right)$ is a homogeneous polynomial of degree $n$.

There is a single marginal solution corresponding to $n=0$ and all the solutions for $n \geqslant 1$ are irrelevant.

Because near $K_{1}=K_{2}=K_{3}=K_{4}=0$, the functions (2.20) are all the homogeneous polynomials of degree $n$, one can consider that arbitrary functions $\left\langle G_{L}\left(K_{1}, K_{2}\right) G_{L}\left(K_{3}, K_{4}\right)\right\rangle$ can be decomposed on the basis of the functions (2.20). The components on the irrelevant $n \geqslant 1$ directions can be forgotten, and one only needs to care about the component $A_{L}$ on the marginal direction. So if the component of $\left\langle G_{L}\left(K_{1}, K_{2}\right) G_{L}\left(K_{3}, K_{4}\right)\right\rangle$ on the marginal direction is

$$
A_{L} \frac{\exp -\left(K_{1}^{2}+K_{3}^{2}\right) / 4-\exp -\left(K_{2}^{2}+K_{4}^{2}\right) / 4}{K_{2}^{2}+K_{4}^{2}-K_{1}^{2}-K_{3}^{2}}
$$


one finds, using (2.19), that $A_{2 L}$ is given by

$$
\frac{A_{2 L}}{4}=\frac{A_{L}}{4}+\frac{\pi^{2}}{2} A_{L}^{2} \iint d q_{1} d q_{2} \frac{\left\{1-\exp \left[-\left(q_{1}^{2}+q_{2}^{2}\right) / 4\right]\right\}^{2}}{\left(q_{1}^{2}+q_{2}^{2}\right)^{2}}
$$

The simplest way of deriving (2.22) is to put $K_{1}=K_{2}=K_{3}=K_{4}=0$ in (2.19) since in that limit the only eigenfunction (2.20) which does not vanish is the marginal eigenfunction.

The integral in (2.22) can be calculated and one ends up with

$$
A_{2 L}=A_{L}+A_{L}^{2} \pi^{3} \log 2
$$

The first thing to notice is that the coefficient of the quadratic term is positive, implying that disorder is marginally relevant. Equation (2.23) can be put in a differential form as

$$
\frac{d A_{L}}{d L}=\pi^{3} \frac{A_{L}^{2}}{L}+\text { higher order terms }
$$

which can be integrated as

$$
-\frac{1}{A_{L}}+\frac{1}{A_{L_{0}}}=\pi^{3}\left(\log L-\log L_{0}\right)
$$

Assume that one starts with a very small. disorder

$$
A_{L_{0}}=\frac{\left\langle b^{2}\right\rangle}{\pi^{4}} \frac{(1+t)^{2}}{(1+2 t) t}
$$

Then (2.25) tells us that the size $L$ at which $A_{L}$ will become of order 1 is

$$
\log L \simeq \frac{\pi}{\left\langle b^{2}\right\rangle} \frac{t(1+2 t)}{(1+t)^{2}}
$$

As seen in Section 2.2, going from the scale $L_{0}$ to the scale $L$, the distance $\Delta a=\langle a\rangle-\left\langle a_{c}\right\rangle^{\text {pure }}$ to the transition of the pure system has been multiplied by $\left(L / L_{0}\right)^{1 / 2}$. Therefore one expects

$$
\log \left(\langle a\rangle_{c}^{\text {random }}-\langle a\rangle_{c}^{\text {pure }}\right) \simeq-\frac{\pi}{2\left\langle b^{2}\right\rangle} \frac{t(1+2 t)}{(1+t)^{2}}
$$

Also from (2.25), we see that the change of scale renormalizes the width of the distribution

$$
A_{L}=\frac{A_{L_{0}}}{1-\pi^{3} A_{L_{0}} \log \left(L / L_{0}\right)}
$$


Replacing $A_{L_{0}}$ by its expression (2.26) and $\sqrt{L}$ by $(\Delta a)^{-1}$, one gets from (2.29) a renormalized width $\left\langle b^{2}\right\rangle_{R}$

$$
\left\langle b^{2}\right\rangle_{R}=\frac{\left\langle b^{2}\right\rangle}{1+\left[2\left\langle b^{2}\right\rangle(1+t)^{2} / \pi t(1+2 t)\right] \log \left(\langle a\rangle-\langle a\rangle_{c}^{\text {pure }}\right)}
$$

In the conclusion, we shall see that this is consistent with the calculation of Forgacs et al., ${ }^{(7)}$ although our interpretation is different.

\section{NUMERICAL RESULTS FOR TWO-DIMENSIONAL WETTING}

In their study of the wetting problem defined by Eqs. (1.1)-(1.3), Forgacs et al. ${ }^{(7)}$ computed the free energy by the transfer-matrix method to test numerically the validity of their results. They found no indication of a shift of the critical temperature nor of a modified critical behavior, in the range of temperatures they could explore. They also noted that large systems (length $L=10^{6}-10^{7}$, width up to 500 ) were necessary to reduce statistical uncertainties to an acceptable level.

We tried to improve this approach by computing also the first two derivatives of the free energy, as in ref. 10: one iterates not only relation (1.3), but also its first and second derivatives. This allows one to study directly the specific heat, even very close to the critical point. We considered specifically the average number of contacts per unit length,

$$
n_{S}=\lim _{L \rightarrow \infty} \frac{1}{L} \frac{d \log Z_{L}}{d \log \langle a\rangle}
$$

and its derivative, the "contact specific heat" $C_{S}$ :

$$
C_{S}=\frac{d n_{s}}{d \log \langle a\rangle}
$$

The number of contacts has the same critical behavior as the energy and it can be obtained directly by differentiating the recursion relations (1.3) with respect to $\langle a\rangle$.

We implemented that approach for a system with rather strong disorder [see (1.5)] $\left(b_{i}= \pm 0.8\right.$ with probability $1 / 2$, and $\left.t=0.25\right)$, on strips of length $L=10^{7}$, discarding the $2 \times 10^{4}$ initial iterations. In these calculations, we also considered an interface between two walls at a distance $2 N$, with the same realization of disorder on the two walls (for symmetry reasons this can be done using tridiagonal transfer matrices of size $N$ ). The sizes studied ranged from $N=25$ to $N=800$. The results in the region very 


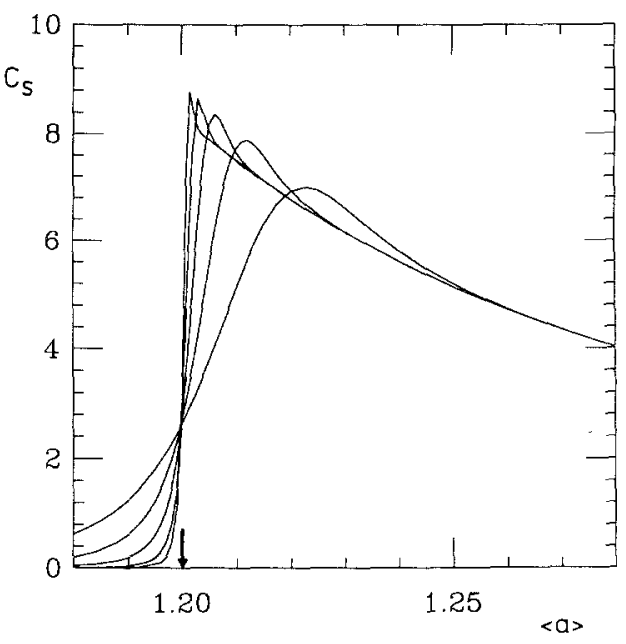

(a)

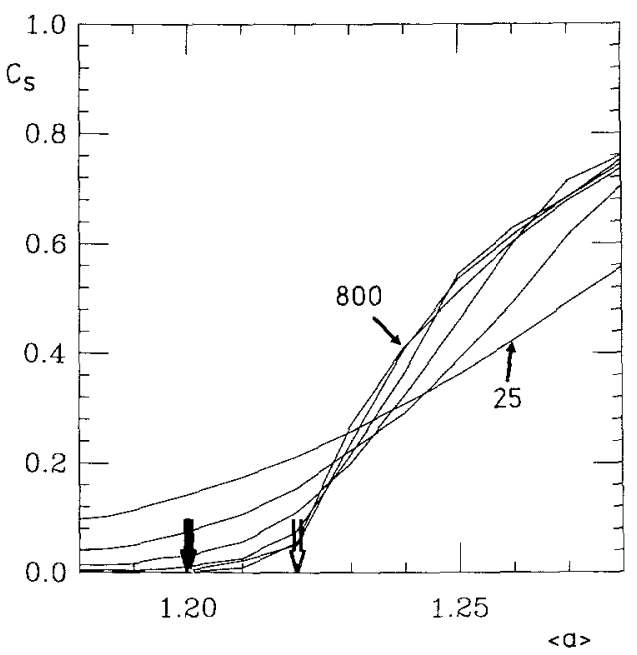

(b)

Fig. 2. (a) Contact specific heat $C_{S}$ versus coupling parameter $\langle a\rangle$, for pure $2 \mathrm{D}$ wetting and system half-widths $N=25,50,100,200$, and 400 . The wetting transition at $\langle a\rangle=1.20$ is indicated by the arrow. (b) Contact specific heat in the presence of disorder (contrast $b=0.8$ ), for strips of length $L=10^{7}$ and strip half-widths $N=25,50,100,200,400$, and 800 . Note the change of vertical scale from part (a). The transition of the pure system is indicated by the black arrow and a rough estimate of the transition in the random system by the white arrow. 
close to $\langle a\rangle_{c}^{\text {pure }}=1.20$ are displayed in Fig. $2 a$ for the pure system and Fig. $2 b$ for the disordered one, to allow a comparison.

In the pure case, the free energy can be obtained from Eq. (A.10) of Appendix A. At the transition point the specific heat $C_{S}$ has a jump from a finite value $C_{S}(\infty)$ to zero, with

$$
C_{S}(\infty)=\frac{2}{t} \frac{(1+t)^{2}}{1+2 t}
$$

A more detailed calculation of finite-size effects, which we shall not describe here, shows that for large $N$ all curves $C_{S}(N)$ versus $\langle a\rangle$ cross at the transition point at a value $C_{S}(\infty) / 3$, in agreement with the results of Fig. 2a.

In presence of disorder (Fig. 2b), the specific heat decreases rapidly to zero with increasing size, when $\langle a\rangle=\langle a\rangle_{c}^{\text {pure }}\left(=\langle a\rangle_{c}^{\text {annealed }}\right)$. There is no sign of a jump. Instead the size dependence of $C_{S}(N)$ strongly suggests that a continuous transition occurs at $\langle a\rangle \simeq 1.22$, with an exponent $\alpha<0$. Our data are too noisy to allow a reliable prediction for $\alpha$, or a study of its dependence on randomness.

Note that for the disorder chosen in (1.5) and (1.6), one can easily prove that $\langle a\rangle_{c}^{\text {random }} \geqslant\langle a\rangle_{c}^{\text {annealed }}$. This is because $\langle\log Z\rangle \leqslant \log \langle Z\rangle$. Since $\langle a\rangle_{c}^{\text {random }}$ is defined by the fact that for $\left.\langle a\rangle\right\rangle\langle a\rangle_{c}^{\text {random }}$, $\langle\log Z\rangle / L>\log (1+2 t)$, it is clear that if the quenched model is in its lowtemperature phase, the same holds for the annealed model.

\section{WETTING ON THE HIERARCHICAL LATTICES}

It is well known that models of statistical mechanics are much easier to solve on hierarchical lattices. ${ }^{(11)}$ The problem then usually reduces to the study of a map in the space of parameters and the critical behavior can be computed by linearizing this map around the critical fixed point. For models with disorder, one has to iterate a whole probability distribution $^{(12-15)}$ and so far there is no analytical approach to calculate the critical behavior (except some perturbative approaches in specific cases ${ }^{(14,15)}$ ).

Here we shall consider the effect of disorder on the wetting transition for a family of hierarchical lattices. The specific heat exponent $\alpha$ of the pure system depends on the branching parameter $B$ of the lattice, and for the particular value of $B$ where $\alpha$ vanishes we shall show that disorder is marginally relevant, as in the $2 \mathrm{D}$ problem studied above. We shall argue similarly that the critical temperature should be shifted by an exponentially small amount in the disorder amplitude, and shall present a numerical study of the nature of the transition. 


\subsection{Uniform Substrate}

The hierarchical lattices we consider are generated by an iterative rule described in Fig. 3: Each bond is replaced by $B$ branches consisting of two bonds, and the construction is repeated. To obtain a model of wetting or, equivalently in the present case, of polymer adsorption, the outer bonds are singled out as wall or "substrate" bonds. An interface (or polymer) extending between the two extremities of the lattice gains an energy $-u_{0}$ every time it lies on the substrate.

The partition function $Y_{k}$ of an interface in the bulk on a $k$ th-generation lattice is given by the recurrence equation

$$
Y_{k+1}=B Y_{k}^{2}
$$

( $Y_{k}$ is just the number of walks, since the energy is zero in the bulk). In the presence of a wall, the branch along the wall differs from the $(B-1)$ bulk branches and the partition function $Z_{k}$ satisfies

$$
Z_{k+1}=Z_{k}^{2}+(B-1) Y_{k}^{2}
$$

The ratio $R_{k}=Z_{k} / Y_{k}$ is thus given by a one-variable recurrence

$$
R_{k+1}=\frac{R_{k}^{2}+(B-1)}{B}
$$

For an initial value $R_{0}=\exp \left(u_{0} / T\right)<B-1, R_{k}$ iterates toward 1 , meaning that on large scales the interface has no tendency to lie close to the wall, it is delocalized, and the system is in its high-temperature phase. For $R_{0}>b-1, R_{k}$ iterates to $\infty$, indicating that the interface is localized close to the wall. The wetting transition occurs at the critical value $R_{0}=B-1$, which is a repulsive fixed point of (4.3).

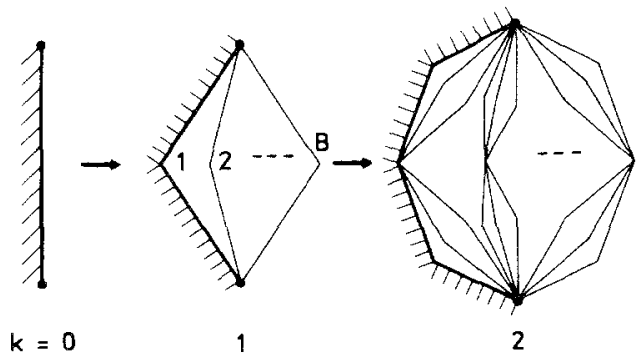

Fig. 3. Recursive construction of a hierarchical lattice with $B$ branches. The hatched surface represents the attractive wall. 
The excess free energy due to the wall

$$
F_{S}=-T \lim _{k \rightarrow \infty} \frac{\log R_{k}}{2^{k}}
$$

vanishes when $R_{0}<B-1$. For $R_{0}>B-1, F_{S}$ has a power law singularity of the form

$$
F_{S} \sim\left[R_{0}-(B-1)\right]^{2-x}
$$

The critical exponent $\alpha$ is obtained as usual by linearizing the recurrence relation (4.3). This gives

$$
R_{k+1}-(B-1) \simeq 2\left(\frac{B-1}{B}\right)\left[R_{k}-(B-1)\right]
$$

Hence

$$
2-\alpha=\frac{\log 2}{\log [2(B-1) / B]}
$$

We note that $\alpha$ vanishes for the particular value of $B$ :

$$
B=2+\sqrt{2}
$$

As we want to compare the hierarchical lattice with the 2D problem of Sections 2 and 3, where disorder is marginal, we will mostly consider the hierarchical lattice for that value of the branching ratio.

\subsection{Random Substrate}

Let us now consider the case where the energies $\left(-u_{i}\right)$ on the substrate bonds are random and uncorrelated. The partition function $Z$ depends on the particular realization of the $\left\{u_{i}\right\}$ and the recurrence equation involves two distinct values $Z^{(1)}$ and $Z^{(2)}$ at the $k$ th generation:

$$
Z_{k+1}=Z_{k}^{(1)} Z_{k}^{(2)}+(B-1) Y_{k}^{2}
$$

Equation (4.3) for the ratio $R$ becomes

$$
R_{k+1}=\frac{R_{k}^{(1)} R_{k}^{(2)}+(B-1)}{B}
$$

with initial conditions $R_{0}^{(i)}=\exp \left(u_{i} / T\right)$. This equation is the direct analogue of $(2.1)$ for the $2 \mathrm{D}$ problem, but it is simpler, as the basic quantity is a number instead of a function. 
Despite its simplicity, this recursion remains nontrivial because, given an initial distribution of the $R_{0}^{(i)}$, it is hard to tell toward what fixed point it will converge.

If the energies are random and uncorrelated, the average $\langle R\rangle$ satisfies the same equation as the pure system (4.3),

$$
\left\langle R_{k+1}\right\rangle=\frac{\left\langle R_{k}\right\rangle^{2}+B-1}{B}
$$

On large scales $\left\langle R_{k}\right\rangle \rightarrow 1$ (respectively, $\infty$ ) if $\left\langle R_{0}\right\rangle\langle B-1$ (resp., $>B-1$ ). If disorder is irrelevant, the wetting transition takes place at the critical point given by

$$
\left\langle R_{0}^{(i)}\right\rangle=B-1=\langle a\rangle_{c}^{\text {pure }}
$$

This occurs if the distribution $P(R)$ becomes narrower under iteration at $T_{c}$. The variance $\Delta=\left\langle R^{2}\right\rangle-\langle R\rangle^{2}$ obeys the recurrence

$$
\Delta_{k+1}=\frac{\Delta_{k}\left(2\left\langle R_{k}\right\rangle^{2}+\Delta_{k}\right)}{B^{2}}
$$

For a weak enough initial disorder, it decreases at $\langle a\rangle=\langle a\rangle_{c}^{\text {pure }}$ if

$$
2\left\langle R_{0}\right\rangle^{2}<B^{2}, \quad \text { that is, } B<2+\sqrt{2}
$$

Comparing this condition with Eq. (4.7), we see that disorder is irrelevant if $\alpha<0$ and relevant if $\alpha>0$, in agreement with the Harris criterion. When $B=2+\sqrt{2}$, the coefficient of $A^{2}$ in (4.13) is positive, implying that disorder is marginally relevant.

It is difficult to elucidate the nature of the transition when disorder is relevant, as the quantity of physical interest is $\langle\log R\rangle$ and its behavior cannot be obtained from that of $\langle R\rangle$ and $\left\langle R^{2}\right\rangle$. As in the 2D case, predictions can be made for the shift of the critical temperature. In the marginal case, for $\langle a\rangle=\langle a\rangle_{c}^{\text {pure }}$, the variance $\Delta_{k}$ grows as

$$
\Delta_{k+1}=\Delta_{k}+\frac{1}{B^{2}} A_{k}^{2}+\cdots
$$

as long as $A_{k} \gg R_{k}-(B-1)$, in direct analogy with Eq. (2.23) for the 2D problem. For small enough $\Delta_{k}$ this yields

$$
\frac{1}{\Delta_{k}} \simeq \frac{1}{\Delta_{0}}-\frac{k}{B^{2}}
$$


where $\Delta_{0}$ is the initial variance, up to a maximum scale $L^{*}$ given by

$$
\log L^{*} \simeq \frac{B^{2} \log 2}{A_{0}}
$$

Following the same reasoning as for the $2 \mathrm{D}$ case, one expects the transition to occur when

$$
\log \left[\left\langle R_{0}\right\rangle-(B-1)\right] \simeq-\frac{B^{2} \log \lambda}{\Delta_{0}}
$$

with $\lambda=2(B-1) / B=\sqrt{2}$. In a model with binary disorder of the form

$$
R_{0}^{(i)}=\langle a\rangle\left(1+b_{i}\right)
$$

with $b_{i}= \pm b$ and $\left\langle b_{i}\right\rangle=0$, the variance is

$$
A_{0}=\langle a\rangle^{2} b^{2}
$$

and one expects the transition point to be shifted for small $b$ by

$$
\log \left(\langle a\rangle_{c}^{\text {random }}-\langle a\rangle_{c}^{\text {pure }}\right) \simeq-\frac{\log 2}{b^{2}}
$$

When disorder is relevant $(B>2+\sqrt{2})$, a calculation along similar lines predicts a power law dependence of the shift:

$$
\left\langle R_{0}\right\rangle-(B-1) \sim \Delta_{0}^{y}
$$

with $y=1 / \alpha$, so the shape of the transition line is controlled by the specific heat exponent of the pure system.

\subsection{Numerical Results}

In order to check that the transition point is indeed shifted in presence of disorder, as predicted from (4.21), and to obtain some information on the nature of the transition, we have performed numerical simulations on hierarchical lattices of large sizes. The randomness was of binary type, as described in (4.19).

4.3.1. Periodic Lattices. One approach consists in determining the critical value of $\langle a\rangle$ for periodic lattices of period $L=2^{k}$. For a given sample this amounts to finding the value $a_{k}^{*}$ of $\langle a\rangle$ such that $R_{k}=B-1$. A distribution $P\left(a_{k}^{*}\right)$ is obtained whose width decreases when $k$ increases. It follows from (4.10) that a system of period $k$ can be critical only if one 
subsystem of size $2^{k-1}$ is below its critical temperature $\left(R_{k-1} \geqslant B-1\right)$ and the other subsystem is above. Hence the average critical coupling $\bar{a}_{k}^{*}$ lies within the range of fluctuation of $a_{k-1}^{*}$.

Results for the marginal branching ratio $B=2+\sqrt{2}$ and a strong contrast $(b=0.8)$ are displayed in Fig. 4 . For $k \geqslant 18$ the critical point of the pure system, $\langle a\rangle_{c}^{\text {pure }}=\sqrt{2}+1$, lies more than two standard deviations away from the average $\bar{a}_{k}^{*}$. This shows that the critical temperature is shifted, albeit by a small amount. Extrapolation of the results yields an estimate for the critical coupling, $\langle a\rangle_{c} \simeq 2.437$. Analogous results were obtained for $b=0.6$, but it was not possible by this approach to study smaller values of $b$ and check the dependence predicted by (4.21) for small $b$.

4.3.2. Finite Lattices. We also studied the average number of contacts per unit length, given here by

$$
n_{S}=\frac{1}{2^{k}} \frac{d\left\langle\log R_{k}\right\rangle}{d \log \langle a\rangle}
$$

and the contact specific heat

$$
C_{S}=\frac{d n_{S}}{d \log \langle a\rangle}
$$

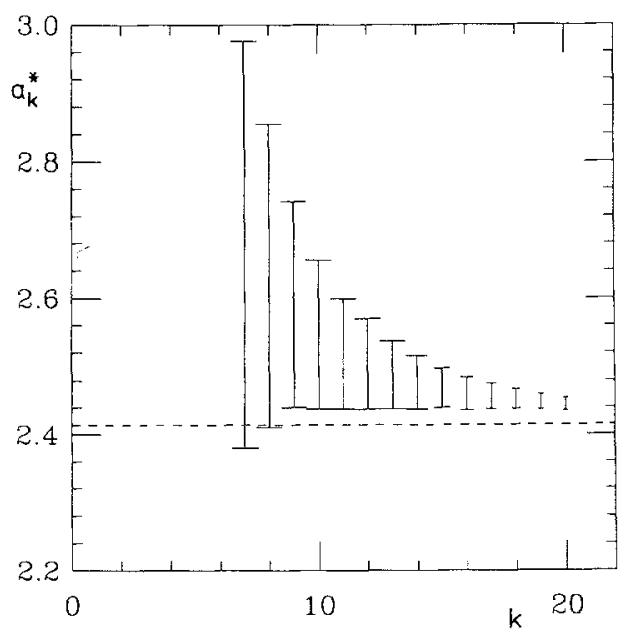

Fig. 4. Critical coupling $a_{k}^{*}$ for periodic hierarchical systems of period $L=2^{k}$ at the marginal branching ratio $B=2+\sqrt{2}$. The intervals correspond to one standard deviation on both sides of the mean value, as obtained from $10^{3}$ samples of length $2^{20}$. The dashed horizontal line indicates the transition of the uniform system. 


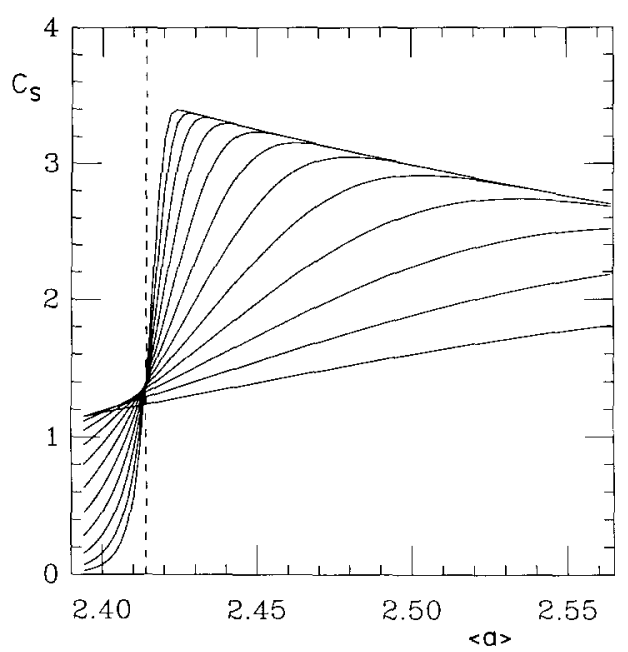

(a)

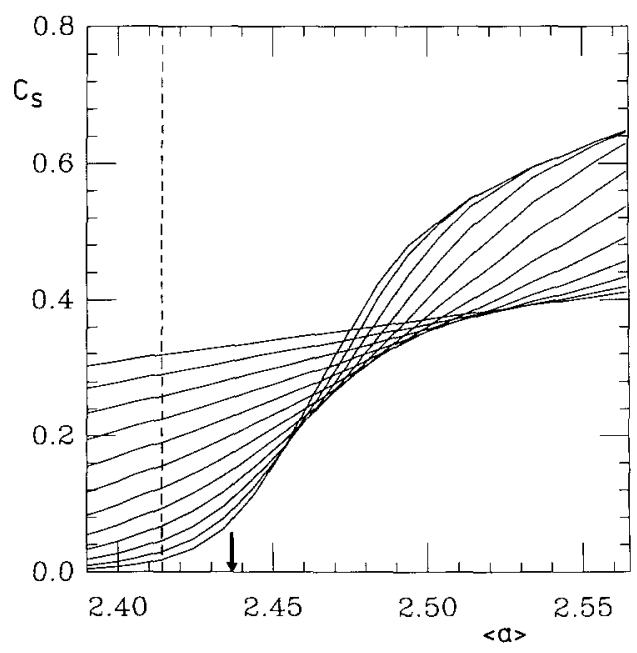

(b)

Fig. 5. (a) Contact specific heat for wetting on a uniform substrate for hierarchical lattices of size $2^{6}-2^{17}$. The vertical dashed line indicates the transition, $\langle a\rangle_{c}=B-1=1+\sqrt{2}$. (b) Contact specific heat for random substrate for hierarchical lattices of size $2^{6}-2^{17}$. The arrow indicates the transition point deduced from the study of periodic systems. The envelope of the curves has the same critical behavior as the specific heat of the infinite system (Appendix B). 
for finite hierarchical lattices of increasing sizes. These quantities can be readily obtained by writing the recurrence relation for the total number of contacts

$$
N_{k+1}=\frac{N_{k}^{(1)}+N_{k}^{(2)}}{1+(B-1) /\left(R_{k}^{(1)} R_{k}^{(2)}\right)}
$$

and the relation for $d N_{k} / d\langle a\rangle$, with initial conditions $N_{0}^{(i)}=1$.

The results obtained in the marginal case $(B=2+\sqrt{2})$ are displayed in Figs. $5 \mathrm{a}$ and $5 \mathrm{~b}$ for the critical region, $\langle a\rangle$ close to $1+\sqrt{2}$. For the pure system the specific heat appears to have a finite jump at the transition point, and the set of curves for different $N$ appear to cross asymptotically at the same point.

In the presence of disorder the behavior is quite different: $C_{S}(k)$ decreases rapidly with system size at the critical point of the pure system, and the results are compatible with a singularity characterized by an exponent $\alpha<0$ at the critical value $\langle a\rangle_{c}^{\text {random }}$ derived by extrapolation of periodic systems.

A more quantitative analysis can be performed by noticing that the envelope $\tilde{C}$ of the set of curves in Fig. $5 \mathrm{~b}$ has the same qualitative behavior as $C_{S}$ for the infinite system, up to a multiplicative constant:

$$
\widetilde{C} \sim K\left(\langle a\rangle-\langle a\rangle_{c}^{\text {random }}\right)^{-\alpha}
$$

This property is discussed in Appendix B. Using the value of $\langle a\rangle_{c}^{\text {random }}$ extracted from the study of periodic systems, one may in this way estimate the specific heat exponent $\alpha$ :

$$
\alpha \simeq-0.6 \text { for } b=0.8
$$

\section{CONCLUSION}

In this paper we have shown (Section 2) that disorder is marginally relevant for the wetting transition in dimension 2. As a consequence, the transition temperature should be shifted and the critical behavior should be changed due to disorder. This is confirmed by our numerical calculations (Section 3) done for a rather strong disorder. As the shift in $\langle a\rangle_{c}$ due to disorder is expected to be exponentially small, the difference between the annealed and the quenched systems becomes quickly invisible when one chooses a weaker disorder. The study of the same wetting problem on a hierarchical lattice leads to very similar conclusions. 
Let us now compare our results with those of previous works. For the same problem, Forgacs et al. ${ }^{(6,7)}$ were able to resum the most singular terms of the weak-disorder expansion. Translating their notations to ours, the result of this resummation was that for $\left\langle b^{2}\right\rangle \ll 1$

$$
\frac{\log Z_{L}}{L}=\log (1+2 t)+\frac{t}{1+2 t} \mu^{2}-\frac{2 \mu^{2}\left\langle b^{2}\right\rangle[(1+t) /(1+2 t)]^{2}}{1-\left\langle b^{2}\right\rangle\left[2(1+t)^{2} / \pi t(1+2 t)\right] \log (1 / \mu)}
$$

where $\mu(>0)$ is small and measures the distance to the critical point $\left\langle a_{c}\right\rangle^{\text {pure }}$ of the pure system,

$$
\langle a\rangle=\frac{1+2 t}{1+t}+\frac{(1+2 t) t}{(1+t)^{2}} \mu
$$

Expression (5.1) is expected to be valid for $\mu$ small but fixed and for infinitesimal $\left\langle b^{2}\right\rangle$.

This expression is consistent with our renormalization calculation of Section 2 as we see in the last term of (5.1) the renormalized width $\left\langle b^{2}\right\rangle_{R}$ obtained in (2.30).

Our conclusions differ, however, from those of Forgacs et al ${ }^{(6,7)}$ in two important aspects. First we find that disorder is marginally relevant and this implies that the critical behavior is changed by disorder, whereas Forgacs et al. claim that it remains unchanged except for a subdominant logarithmic correction. Their conclusion would be reached by taking the limit $\mu \rightarrow 0$ in formula (5.1) without worrying about the fact that the denominator vanishes when $\mu$ decreases, meaning that the perturbation expansion loses its validity. Second, we conclude that for any amount of disorder, the quenched and the annealed critical $\langle a\rangle$ are different, in agreement with our numerical results.

At the moment, predicting the nature of the transition in the presence of disorder, even in the simple case of the hierarchical lattice, remains a challenging open question.

\section{APPENDIX A. PARTITION FUNCTION OF THE PURE SYSTEM AT THE WETTING POINT IN DIMENSION 2}

In this appendix, we show that the partition function of the pure system is given by formula (2.6) at the wetting transition of the pure system, while at infinite temperature (high-temperature fixed point) it is given by 


$$
\begin{aligned}
Z_{L}^{\mathrm{HT}}(x, y) \sim & (1+2 t)^{L}\left(\frac{1+2 t}{4 \pi t L}\right)^{1 / 2}\left\{\exp \left[-\frac{(x-y)^{2}(1+2 t)}{4 t L}\right]\right. \\
& \left.-\exp \left[-\frac{(x+y)^{2}(1+2 t)}{4 t L}\right]\right\}
\end{aligned}
$$

$Z_{L}(x, y)$ can be interpreted as the partition function of directed random walks of $L$ steps going from point $(0, x)$ to point $(L, y)$. Each step of a walk is either horizontal with weight 1 or along one of the diagonals of the lattice with weight $t$. Moreover, the sites visited by the walks are constrained to have positive ordinates and each contact with the wall contributes an additional weight $a$.

Let us first consider unconstrained walks. Then the partition function $Z_{L}^{\mathrm{RW}}(x, y)$ of the walks going from $(0, x)$ to $(L, y)$ in $L$ steps is simply the coefficient of $u^{y-x}$ in the polynomial $[1+t(u+1 / u)]^{L}$. This can be written

$$
Z_{L}^{\mathrm{RW}}(x, y)=\oint \frac{d u}{2 \pi i} \frac{1}{u^{y-x+1}}\left[1+t\left(u+\frac{1}{u}\right)\right]^{L}
$$

where the integral is taken around the unit circle in the complex $u$ plane. At infinite temperature, $a$ is equal to one. Therefore one can take the constraint into account by the method of images

$$
Z_{L}^{\mathrm{HT}}(x, y)=Z_{L}^{\mathrm{RW}}(x, y)-Z_{L}^{\mathrm{RW}}(x,-y-2)
$$

$Z_{L}^{\mathrm{RW}}(x, y)$ for large $L$ and $x$ or $y$ of order $\sqrt{L}$ can be estimated by using the integral representation (A.2) and the saddle point method. One obtains

$$
Z_{L}^{\mathrm{RW}}(x, y) \simeq(1+2 t)^{L}\left(\frac{1+2 t}{4 \pi t L}\right)^{1 / 2} \exp \left[-\frac{(x-y)^{2}(1+2 t)}{4 t L}\right]
$$

By substituting (A.4) in formula (A.3), one gets formula (A.1) for the hightemperature fixed point.

Let us now consider the computation of $Z_{L}(x, y)$ at the wetting transition. It is convenient to decompose the walks into two classes: (i) walks that never touch the wall, and (ii) walks that have at least one point of contact with the wall.

The contribution of the first class is obtained easily by the method of images,

$$
Z_{L}^{(\mathrm{i})}(x, y)=Z_{L}^{\mathrm{RW}}(x, y)-Z_{L}^{\mathrm{RW}}(x,-y)
$$

In the asymptotic limit $(L \gg 1$ and $x, y \sim \sqrt{L}), Z_{L}^{(\mathrm{i})}(x, y)$ is given by formula (A.1) as $Z_{L}^{\mathrm{HT}}(x, y)$. 
Each walk of the second class can be decomposed into three parts: (a) a random walk between $(0, x)$ and the first point of contact with the wall; (b) a random walk between the last point of contact with the wall and the point $(L, y)$; and (c) a middle part of the walk between the first and last contacts with the wall with an arbitrary number of contacts in between.

The contributions coming from the first part and last part of the walks are easily evaluated. They are related to random walks that are shorter by one step and never touch the wall. They can be computed using the partition function $Z^{(\mathrm{i})}$ introduced above. Therefore $Z_{L}^{(\mathrm{ii})}(x, y)$ can be written

$$
Z_{L}^{(\mathrm{ii})}(x, y)=\sum_{L_{1}+L_{2}+L_{3}=L} t Z_{L_{1}-1}^{(\mathrm{i})}(x, 1) a Z_{L_{2}}^{(\mathrm{ii})}(0,0) t \dot{Z}_{L_{3}-1}^{(\mathrm{i})}(1, y)
$$

Notice that in $Z^{(\mathrm{i})}$ the weight coming from the contact with the wall does not appear, while in $Z_{L}^{(i i)}$ we count the weight from abscissa 1 to abscissa $L$. This is why only the leftmost weight in $Z_{L_{2}}^{(\text {ii) }}$ appears explicitly in formula (A.6). The new piece in formula (A.6) is $Z_{L_{2}}^{(\mathrm{ii})}(0,0)$, which comes from the central part of the walk. In order to compute it, we write a recursion relation which relates it to walks with one less contact point,

$$
Z_{L}^{(i i)}(0,0)=a Z_{L-1}^{(\mathrm{ii})}(0,0)+\sum_{L^{\prime}=2}^{L} a t^{2} Z_{L^{\prime}-2}^{(\mathrm{i})}(1,1) Z_{L-L^{\prime}}^{(\mathrm{ii})}(0,0)
$$

This can be solved by introducing generating functions

$$
\begin{aligned}
& \tilde{Z}_{\mu}^{(\mathrm{ii})}(0,0)=\sum_{L=0}^{\infty} \mu^{L} Z_{L}^{(\mathrm{ii})}(0,0) \\
& \tilde{Z}_{\mu}^{(\mathrm{i})}(1,1)=\sum_{L=0}^{\infty} \mu^{L} Z_{L}^{(\mathrm{i})}(1,1)=\frac{1}{2 \mu^{2} t^{2}}\left\{1-\mu-\left[(1-\mu)^{2}-4 \mu^{2} t^{2}\right]^{1 / 2}\right\}
\end{aligned}
$$

Then (A.7) reads

$$
\tilde{Z}_{\mu}^{(\mathrm{ii})}(0,0)-1=\mu a \widetilde{Z}_{\mu}^{(\mathrm{ii})}(0,0)+a \mu^{2} t^{2} \widetilde{Z}_{\mu}^{(\mathrm{i})}(1,1) \tilde{Z}_{\mu}^{(\mathrm{ii})}(0,0)
$$

and

$$
\tilde{Z}_{\mu}^{(\mathrm{ii})}(0,0)=\left\{1-\frac{a}{2}-\frac{\mu a}{2}+\frac{a}{2}\left[(1-\mu)^{2}-4 \mu^{2} t^{2}\right]^{1 / 2}\right\}^{-1}
$$

The behavior of $Z_{L}^{(\mathrm{ii})}(0,0)$ is determined by the singularity of $\tilde{Z}_{\mu}^{(\mathrm{ii})}(0,0)$ closest to the origin $\mu=0$. For $a$ small, it comes from the square root and stands at $\mu^{*}=(1+2 t)^{-1}$. This corresponds to the high-temperature phase. On the contrary, for $a$ sufficiently large, the singularity closest to $\mu=0$ is 
a pole coming from the zero of the denominator in (A.10). This describes the low-temperature dry phase. The transition point between the two behaviors is given by the coincidence of the two singularities, that is, for $a_{c}$ such that the denominator vanishes at $\mu=\mu^{*}$. This gives the wetting transition point $a_{c}=(1+2 t) /(1+t)$, which is easily interpreted as the point where the loss of entropy due to the presence of the wall is exactly counterbalanced by the gain of energy at the contact point. At the wetting transition point the behavior of $\tilde{Z}_{\mu}^{(\mathrm{ii})}(0,0)$ is given by

$$
\tilde{Z}_{\mu}^{(i i)}(0,0) \sim\left[\frac{1}{t(1+2 t)}\right]^{1 / 2} \frac{1+t}{\left(1-\mu / \mu^{*}\right)^{1 / 2}}
$$

and therefore

$$
Z_{L}^{(i i)}(0,0) \sim \frac{1+t}{[t(1+2 t) \pi L]^{1 / 2}}(1+2 t)^{L}
$$

In order to compute $Z_{L}^{(\mathrm{ii})}(x, y)$ in the limit $L \gg 1, x \sim L^{1 / 2}, y \sim L^{1 / 2}$, we also need the asymptotic behavior of $Z_{L}^{(i)}(x, 1)$ and $Z_{L}^{(i)}(1, y)$. From (A.5) and (A.4) one obtains

$t Z_{L-1}^{(\mathrm{i})}(x, 1)=t Z_{L-1}^{(\mathrm{i})}(1, x) \simeq(1+2 t)^{L}\left(\frac{1+2 t}{4 \pi t L}\right)^{1 / 2} \frac{x}{L^{3 / 2}} \exp \left[-\frac{x^{2}}{4 t L}(1+2 t)\right]$

The contribution of walks that have at least one contact point with the wall can now be obtained by substituting (A.12) and (A.13) in (A.6) and by replacing the sum by an integral. One gets

$$
\begin{aligned}
Z_{L}^{(i i)}(x, y) \simeq & \frac{(1+2 t)^{L}}{4}\left(\frac{1+2 t}{\pi t}\right)^{3 / 2} x y \int_{0}^{L} d L^{\prime} \int_{0}^{L-L^{\prime}} d L^{\prime \prime} \\
& \times \frac{\exp \left\{-[(1+2 t) / 4 t]\left(x^{2} / L^{\prime}+y^{2} / L^{\prime \prime}\right)\right\}}{\left(L^{\prime} L^{\prime \prime}\right)^{3 / 2}\left(L-L^{\prime}-L^{\prime \prime}\right)^{1 / 2}}
\end{aligned}
$$

This expression can be simplified by using the result

$$
\int_{0}^{1} d \alpha \int_{0}^{1-\alpha} d \beta \frac{\exp \left(-u^{2} / \alpha-v^{2} / \beta\right)}{(\alpha \beta)^{3 / 2}(1-\alpha-\beta)^{1 / 2}}=\frac{\pi}{|u v|} \exp \left[-(u+v)^{2}\right]
$$

Finally, one obtains the simple formula

$$
Z_{L}^{(\mathrm{ii})}(x, y) \sim(1+2 t)^{L}\left(\frac{1+2 t}{\pi t L}\right)^{1 / 2} \exp \left[-\frac{(1+2 t)}{4 t L}(x+y)^{2}\right]
$$


The expression of $Z_{L}(x, y)$ at the wetting transition critical fixed point is obtained by adding the contributions of $Z_{L}^{(i)}(x, y)$ and $Z_{L}^{(i i)}(x, y)$. One gets, in agreement with Eq. (2.6),

$$
\begin{aligned}
Z_{L}(x, y)= & (1+2 t)^{L}\left(\frac{1+2 t}{4 \pi t L}\right)^{1 / 2}\left\{\exp \left[-\frac{1+2 t}{4 t L}(x-y)^{2}\right]\right. \\
& \left.+\exp \left[-\frac{1+2 t}{4 t L}(x+y)^{2}\right]\right\}
\end{aligned}
$$

As a final remark, one can note that at the wetting transition, $Z_{L}^{(i i)}(0,0)$ decreases like $L^{-1 / 2}$. Using (A.12), this can be the starting point of a conventional renormalization group treatment in the replica formalism to show that disorder is marginally relevant and that the two-replica interaction is the only important one. In this way, one can obtain an alternative derivation of formula (2.30) of the main text, from the computation of a one-loop, two-vertex graph.

\section{APPENDIX B. CRITICAL BEHAVIOR OF THE ENVELOPE FUNCTION}

We show here that the critical behavior of a quantity $C(T)$ singular at $T=T_{c}$ can be obtained from the envelope of the curves $C_{N}(T)$ for finite system sizes.

Suppose that close to $T_{c}, C(T)$ has a singularity of the form

$$
C(T) \sim\left|T-T_{c}\right|^{\varphi}
$$

and that for large system sizes, it satisfies a finite-size scaling relation of the form

$$
C_{N}(T) \simeq N^{-\varphi / v} f\left[\tau N^{1 / v}\right]
$$

where $f(z)$ is a smooth function for $|z| \ll 1, \tau=T-T_{c}$, and $v$ is the correlation length exponent. If an envelope $\widetilde{C}(T)$ exists for the curves $C_{N}(T)$, it is defined by

$$
\widetilde{C}(T)=C_{N_{0}}(T)
$$

where $N_{0}(T)$ is the size where $C_{N_{0}+1}(T)=C_{N_{0}}(T)$. This is the solution of

$$
\left.\frac{\partial C_{N}}{\partial N}\right|_{N_{0}}=0
$$


Hence $z_{0}=\tau N_{0}^{1 / v}$ is the solution (if it exists) of

$$
-\varphi f\left(z_{0}\right)+z_{0} f^{\prime}\left(z_{0}\right)=0
$$

The envelope is given by

$$
\widetilde{C}(T) \simeq K \tau^{\varphi}
$$

where $K=z_{0}^{-\varphi} f\left(z_{0}\right)$ is a constant independent of size. Thus $\tilde{C}(T)$ has the same critical behavior as $C(T)$. The envelope may reduce to a point if $\varphi=0$, as in the pure $2 \mathrm{D}$ wetting problem.

A useful consequence of (B.6) is that the critical point can be located graphically with reasonable accuracy if $\varphi \leqslant 1$, whereas the usual scaling analysis involves three parameters $\left(T_{c}, v\right.$, and $\left.\varphi\right)$ which have to be fitted at the same time.

\section{ACKNOWLEDGMENTS}

We thank J. P. Bouchaud, M. Gingras, J. M. Luck, and H. Orland for useful comments.

\section{REFERENCES}

1. A. B. Harris, J. Phys. C 7:1671 (1974).

2. G. Grinstein, in Fundamental Problems in Statistical Mechanics VI, E. G. D. Cohen, ed. (1985), p. 147.

3. D. B. Abraham, in Phase Transitions and Critical Phenomena, Vol, 10, C. Domb and J. L. Lebowitz, eds. (1986), p. 1.

4. H. W. Diehl, in Phase Transitions and Critical Phenomena, Vol.10, C. Domb and J. L. Lebowitz, eds. (1986), p. 75.

5. M. Schick, in Les Houches, XLVIII Liquids at Interfaces, J. Charvolin, J. F. Joanny, and J. Zinn-Justin, eds. (1990), p. 415.

6. G. Forgacs, J. M. Luck, Th. M. Nieuwenhuizen, and H. Orland, Phys. Rev. Lett. 57:2184 (1986).

7. G. Forgacs, J. M. Luck, Th. M. Nieuwenhuizen, and H. Orland, J. Stat. Phys. 51:29 (1988).

8. V. S. Dotsenko and Vl. S. Dotsenko, Adv. Phys. 32:129 (1983); R. Shankar, Phys. Rev. Lett. 58:2466 (1987).

9. J. S. Wang, W. Selke, Vl. S. Dotsenko, and V. B. Andreichenko, Physica A 16:221 (1990).

10. B. Derrida and O. Golinelli, Phys. Rev. A 41:4160 (1990).

11. A. N. Berker and S. Ostlund, J. Phys. C 12:4961 (1979).

12. W. Kinzel and E. Domany, Phys. Rev. B 23:3421 (1981).

13. D. Andelman and A. N. Berker, Phys. Rev. B 29:2630 (1984).

14. B. Derrida and E. Gardner, J. Phys. A 17:3223 (1984).

15. B. Derrida and R. B. Griffiths, Europhys. Lett. 8:111 (1989); J. Cook and B. Derrida, J. Stat. Phys. 57:89 (1989). 